\title{
Success rates of molar apicectomy with amalgam root-end filling
}

\author{
Molar apicectomy with amalgam root-end filling: results of a prospective study in two district general hospitals \\ C. M. Wesson and T. M. Gale Br Dent J 2003; 195: 707-714
}

\section{Aim}

To determine the five-year success rates, site or sites of failure, prognostic indicators and lower lip morbidity associated with molar apicectomy using amalgam root-end filling.

\section{Design and Setting}

Multicentre, prospective study. The departments of oral and maxillo-facial surgery in two district general hospitals.

\section{Method}

During 1974-1995, 1,007 molar apicectomy procedures, combined with amalgam root-end filling were expedited. A five-year review of each tooth was carried out or attempted between 1979-2000.

\section{Results}

Of the $790(78 \%)$ operated molars successfully reviewed at 5 years or later, 451 (57\%) exhibited 'complete healing' and 39 (5\%) 'uncertain healing. Three hundred (38\%) were classified as 'unsatisfactory healing' (failures), including 12 which were assumed to be of periodontal origin. Mandibular first molars had the highest "complete healing' rate $(60 \%)$ and mandibular second molars the lowest (46\%). Teeth which showed 'complete healing' at 1 year had a 75\% probability of maintaining this outcome at 5 years. Sensory disturbance of variable duration occurred in the lower lip following 20$21 \%$ of mandibular molar procedures. In the majority of cases (79-80\%) this had remitted within 3 months. A permanent deficit occurred in 8 patients (1\%) where the apicectomy could definitely be incriminated as causative. Four were associated with first molar apicectomy and four with second molar apicectomy.

\section{Conclusions}

Molar apicectomy with amalgam root-end filling attracts an overall 'complete healing' rate at 5 years of $57 \%$, the results being best with mandibular first molars and worst with mandibular second molars. The prognosis is also better where there is 'good' initial orthograde root filling, an associated radicular cyst as compared with granulomatous change and where the buccal sulcus is deep rather than shallow. It is worse when orthograde root filling is absent and when there is disease in the furcation. 'Complete healing' at 1 year can be expected to be maintained at 5 years in 75\% of cases. There is a threefold increase in the occurrence of permanent lower lip sensory impairment following second molar surgery in comparison with first molar surgery, the overall incidence being $1 \%$.

\section{IN BRIEF}

- Molar apicectomy with amalgam root-end filling attracted an overall 5-year 'complete healing' rate of 57\%.

- The apical ends of the roots were commoner sites of failure than the furcation. Purely lateral failure was not seen.

- The significant complication was impaired sensation in the lower lip following mandibular molar apicectomy. This occurred for a variable period in $20-21 \%$ of cases. A permanent deficit followed in $1 \%$.

- 'Complete healing' at 1 year was maintained in $75 \%$ of cases at 5 years.

\section{COMMENT}

This study has almost been a lifetime's work, starting in 1974, when molar apicectomy in the UK was a novelty. The first author pioneered saving molars, when many others would have removed the teeth. He had the foresight to plan a prospective study and to carry it through to completion, when he probably had many other demands on his time. Had he been able to complete it 10 years earlier, it would have been regarded as a significant contribution to the evidence base of current practice. However, time does not stand still, and the last decade has seen major changes in the practice of root-end surgery. There has been the introduction of surgical loupes, or microscopes, the use of ultrasonic instrumentation for root-end preparation, the avoidance of a root-face bevel, special intrabony mirrors and a range of root-end filling materials. The use of amalgam for root-end filling has ceased, and a recent prospective study by Chong etal. ${ }^{1}$ has found a 2-year success rate using alternative materials close to $90 \%$.

This study will be seen as reporting the outcome of a historical procedure, but that should not detract from its value. It reports on molar teeth, which were largely omitted in previous studies. The findings are in line with those reported by Friedman, ${ }^{2}$ but they do dispel the myth that surgery has a lower outcome in molars.

As a dental public health procedure, admitting patients to hospital to manage apical periodontitis is an inappropriate way to treat patients and use public resource. Most of these patients could be managed by root canal retreatment performed by endodontic specialists in outpatient clinics, where the outcome would be better, the morbidity to patients lower and the cost less. It is of concern that 5 years after the specialist list in endodontics came into existence, there is no NHS funded training in England nor NHS clinics where this type of treatment can be obtained.

\section{T. R. Pitt Ford, Professor of Endodontology}

GKT Dental Institute, King's College London

1. Chong B S, Pitt Ford T R, Hudson M B. A prospective clinical study of mineral trioxide aggregate and IRM when used as root-end filling materials in endodontic surgery. Int End J 2003; 36: 520-526.

2. Friedman S. Treatment outcome and prognosis of endodontic therapy. In: Orstavik D, Pitt Ford TR (Eds). Essential endodontology: prevention and treatment of apical periodontitis. Oxford: Blackwell Science, 1998 pp 367-401.

doi:10.1038/sj.bdj.4810830 